\title{
FMTVDM/BESTC (P) Imaging for Women with Breast Implants and Dense Breasts
}

\author{
Richard M Fleming, PhD, MD, JD', Matthew R Fleming, BS ${ }^{1}$, Tapan K Chaudhuri, MD', William C \\ Dooley, MD', and Andrew Mc Kusick, BS Pharm ${ }^{1,4}$
}

${ }^{1}$ FHHI-Omnificlmaging-Camelot, Los Angeles, CA, USA

${ }^{2}$ Eastern Virginia Medical School, USA

${ }^{3}$ Oklahoma University Health Science Center, USA

${ }^{4}$ Sebec Consulting \& Media, USA

*Corresponding author: Richard M Fleming, PhD, MD, JD, FHHI-Omnific Imaging-Camelot, Los Angeles, CA, USA

\section{Keywords}

FMTVDMC®, BEST imaging@®, Breast cancer, Breast inflammation, Theranostics, Quantification, Al, Nuclear camera quantitative calibration, Patent protected, Breast implants, Dense breasts

Prior to FMTVDM/BEST Imaging, diagnostic testing was limited to looking for breast cancer either using "qualitative" imaging defining disease as either present or absent, or semiquantitative methods [1-10] which is also limited to the same yes you have breast cancer or no you don't interpretation. These imaging methods include mammography, ultrasound, CT, MRI, as well as other SPECT/Planar and PET imaging approaches and as such are with associated sensitivity and specificity problems. These tests are further limited when imaging dense breast tissue or when breast implants exist either for augmentation or following mastectomy.

The ability of FMTVDM/BEST to differentiate transitional changes in tissue, associated with regional blood flow differences (RBFDs) and increasing tissue metabolism, makes it possible for FMTVDM/BEST to measure these tissue changes across an entire spectrum of changes; recognizing a "Health-Spectrum" shown by Figure 1. The interaction between cellular genetics and environmental factors consequently influence changes in tissue resulting in transitional changes including regional blood flow differences and metabolism. At one end of the spectrum is the absence of life, which may or may not be associated with a significant accumulation of cellular debris, inter alia, calcium, which may occur prior to the actual loss of cellular "life". In order of increasing regional blood flow and cellular metabolism are what is considered to be the "normal" state of a given tissue; resulting changes occurring due to an increased signaling and consequential accumulation of an inflammatory state; sequentially followed by further changes in tissue transitioning through increasingly metabolically active tissue with increased metabolic activity and demands for increased regional blood flow, until arriving at a tissue state where the expected controls and functionality of tissue no longer exist; what has classically been defined as "cancer". Implicit in this understanding is the appreciation that this continuum across transitional states reflects the interrelationship between these various "Health-Spectrum" states, which (1) Are not defined by some arbitrary absolute cutoffs between tissue states, (2) Demonstrate the ability of tissues to transition in either direction towards "normalization" or towards "cancer" and (3) Allow for the absolute measurement of the affect of treatment upon any of these tissue states across the "Health-Spectrum" providing the ability to direct care at the "patient-specific" level, improving outcomes across these transitional states; saving time, money and most importantly lives.

Citation: Fleming RM, Fleming MR, Chaudhuri TK, Dooley WC, Mc Kusick A (2019) FMTVDM/BEST@ @ Imaging for Women with Breast Implants and Dense Breasts. Int J Womens Health Wellness 5:092. doi. org/10.23937/2474-1353/1510092

Accepted: April 06, 2019: Published: April 08, 2019

Copyright: (c) 2019 Fleming RM, et al. This is an open-access article distributed under the terms of the Creative Commons Attribution License, which permits unrestricted use, distribution, and reproduction in any medium, provided the original author and source are credited. 
Measuring changes in breast tissue as it changes from normal to cancer.
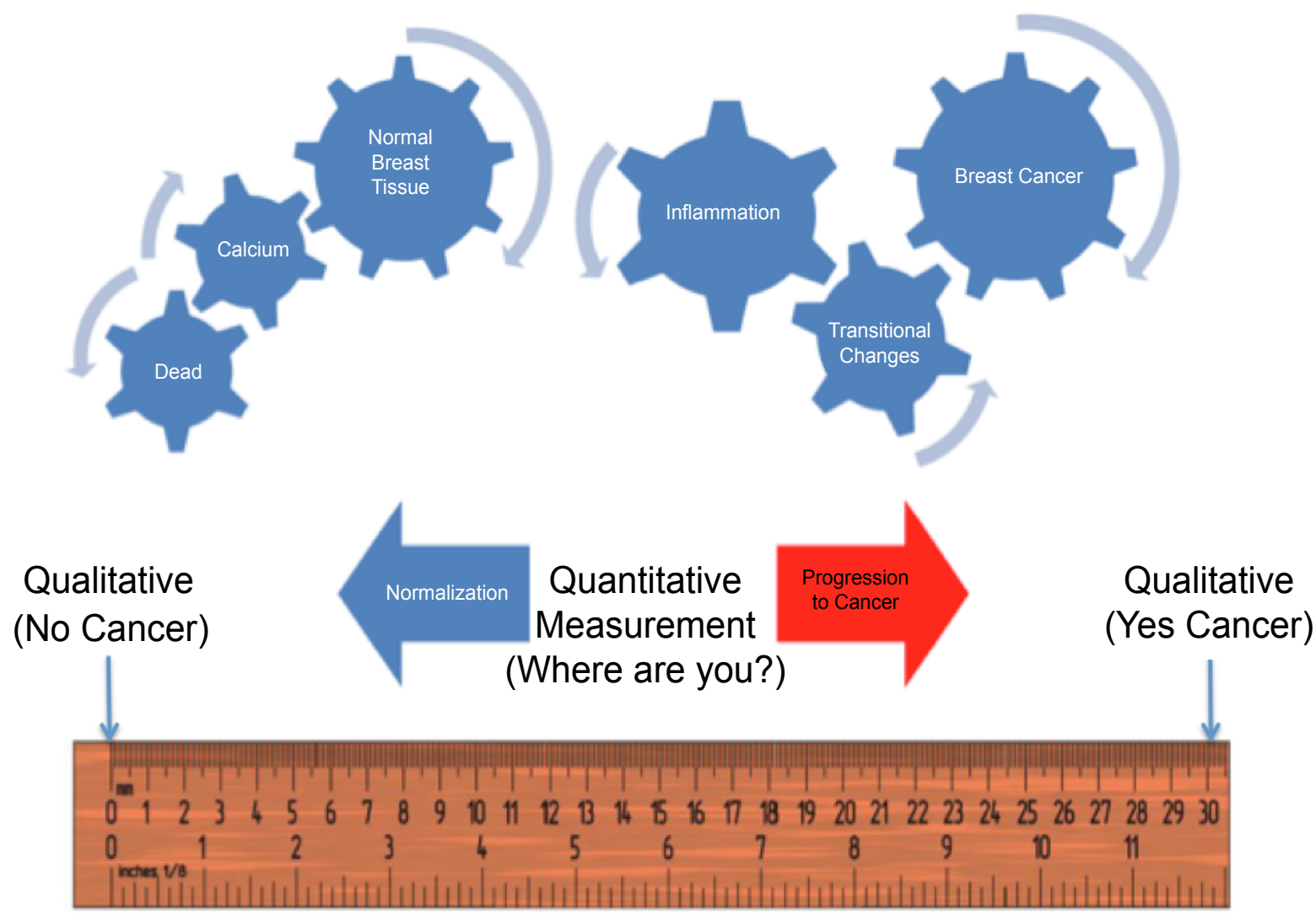

Figure 1: FMTVDM/BEST "Health-Spectrum" Measurements.

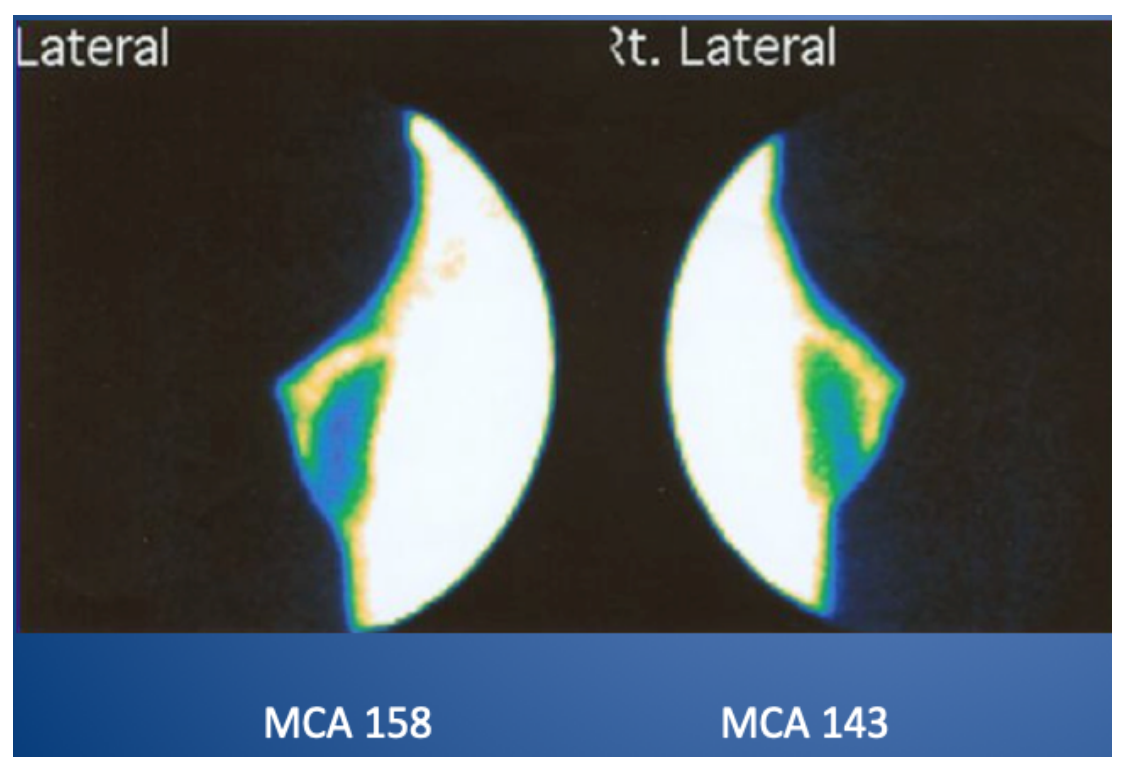

Figure 2: FMTVDM/BEST Imaging of a woman who has undergone bilateral prophylactic mastectomy and breast implants after testing positive for BRCA1/2.

Because FMTVDM/BEST measures the RBFDs and metabolism of tissue, which is not altered by dense breasts or foreign bodies; e.g. breast implants, FMTVDM/BEST can be used to evaluate the "health" of a woman's breast in such circumstances. The following study represents a woman who has breast implants evaluated using FMTVDM/BEST.
FMTVDM/BEST Imaging of Woman with Bilateral Breast Implants and Compressed (Dense) Breasts

A 38-year-old woman positive for BRCA1 \& 2 who had previously undergone bilateral prophylactic mastectomy followed by bilateral breast implants was referred for diagnostic evaluation following mammographic 
questions associated with dense breasts from the residually compressed breast tissue in addition to limitations associated with breast implants.

She underwent FMTVDM/BEST imaging per protocol [11] with the measurements shown in Figure 2, which shows the lateral image of the left breast followed by the lateral image of the right breast. FMTVDM/BEST Imaging of woman with bilateral breast implants. Breast implants are shown in "blue" with compression of breast tissue (yellow) showing the left breast to the left and the right breast to the right in these images. The measured results reveal counts of 158 in the left breast and 143 in the right breast, both associated with "normal" breast tissue with early "inflammatory" changes. In both instances, the appearance of the breast tissue (yellow) appears to be visually increased particularly when compared with the breast implants, which are blue in appearance surrounded by less compressed breast tissue, noted in green.

Despite the appearance, FMTVDM/BEST measurements placed the woman in the upper range for "normal" breast tissue approaching "inflammatory" changes, measuring 158 in the left breast and 143 in the right.

\section{Discussion}

The limitations in mammography for women with dense breasts has resulted in legislation existing in more than 30 U.S. States, requiring the inclusion of statements with the mammography report notifying physicians and women that mammograms may miss breast cancer in women with dense breasts. In women with genetic markers associated with an increased risk for breast cancer many have elected to undergo bilateral prophylactic mastectomy to reduce their risk of breast cancer. While this may reduce the potential for breast cancer it does not altogether eliminate it and the inclusion of breast implants now adds an additional complicating factor into breast imaging.

FMTVDM/BEST Imaging provides the only quantitative method for measuring changes in RBFDs and metabolic tissue differences across the "Health-Spectrum". While the qualitative imagine may appear abnormal, the true measurement of tissue health with FMTVDM/ BEST allows clinicians to be able to monitor women's breast health in the presence of dense breasts and breast implants.

\section{References}

1. Fleming RM, Fleming MR, Dooley WC, Sheikh A, McKusick A, et al. (2018) FMTVDM -FHRWW \& BESTCP The first True "Quantitative" nuclear imaging protocols with proprietary equations following The Fleming Method (TFM) for nuclear scintillation equipment quantitative standardization. Biomed J Sci \& Tech Res 4: 1-4.

2. Fleming RM, Fleming MR, McKusick A, Chaudhuri $T$ (2018) FMTVDM-TFMOP: True quantification requires standardization of the tool being used to measure, with a known, unchanging standard to produce accurate, consistent and reproducible quantified measurements. J Nucl Card 2018.

3. Fleming RM, Fleming MR, McKusick A, Chaudhuri TK, Dooley WC, et al. (2018) FMTVDMCP stress-first/stressonly imaging is here! But first we need to clarify the use of what (1) Stress, (2) Rest, (3) Redistribution and (4) Quantification, really mean. J Nucl Med Radiat Ther 9.

4. Fleming RM, Fleming MR, McKusick A, Chaudhuri TK (2018) Semi-quantification limitations: FMTVDMC) demonstrates quantified tumor response to treatment with both regional blood flow and metabolic changes. $J$ Nucl Med 59: 1643-1644.

5. Fleming RM, Fleming MR, McKusick A, Chaudhuri TK (2018) FMTVDMO@ Nuclear Imaging Artificial (Al) Intelligence but fires we need to clarify the use of (1) Stress, (2) Rest, (3) Redistribution and (4) Quantification. Biomed J Sci \& Tech Res 7: 1-4

6. Fleming RM, Fleming MR, McKusick A, Chaudhuri TK (2018) Virtual quantification is not True quantification. FMTVDM-TFMCP provides true quantification for SPECT and PET. Archives of Medicine 10: 7.

7. Sheikh A (2018) Evolution of quantification in clinical nuclear medicine: A brief overview of salient uses and upcoming trends. J Nucl Med Radiat Ther 9: 4.

8. Fleming RM (2018) FMTVDMOP provides the first true spect and pet quantification and not virtual guesstimation produced by SUV and extraction fraction, yielding first accurate theranostic method. J Nucl Med Radiat Ther 9: 4.

9. Fleming RM, Fleming MR, Dooley WC, McKusick A, Chaudhuri T (2018) FMTVDMC® provides the first nuclear quantitative method for nuclear cardiology and introduces a new era for nuclear cardiology. J Nucl Card 25: 1453.

10. Fleming RM, Fleming MR, Chaudhuri TK, Dooley WC, McKusick A (2019) Letter to the editor: A response to Hruska's case study on molecular breast imaging and the need for true tissue quantification. Breast Cancer Res 21: 15.

11. Rm Fleming The Fleming Method for Tissue and Vascular Differentiation and Metabolism (FMTVDM) using same state single or sequential quantification comparisons. Patent Number 9566037. 